\title{
ARTICLE
}

Cite this: DOI: 10.1039/xoxxooooox

Received ooth January 2012, Accepted ooth January 2012

DOI: $10.1039 /$ xoxxooooox

www.rsc.org/

\section{Effect of Oxidative Treatment on the Secondary Structure of Decoloured Bloodmeal}

\author{
Talia Hicks ${ }^{a}$, Casparus J. R. Verbeek ${ }^{a}$, Mark C. Lay a and James Bier ${ }^{a}$
}

\begin{abstract}
Bloodmeal can be decoloured using peracetic acid resulting in a material with a pale-yellow colour which only needs sodium dodecyl sulphate, water and triethylene glycol to extrude into a semitransparent bioplastic. Fourier-transform infrared (FTIR) spectroscopy using Synchrotron light was used to investigate the effect of peracetic acid treatment at various concentrations on the spatial distribution of secondary structures within particles of bloodmeal. Oxidation caused aggregation of helical structures into sheets and acetic acid suppressed sheet formation. Decolouring with peracetic acid led to particles with a higher degree of disorder at the outer edges and higher proportions of ordered structures at the core, consistent with the expected diffusion controlled heterogeneous phase decolouring reaction. The degradation of stabilizing intra- and intermolecular interactions and the presence of acetate ions results in increased chain mobility and greater amorphous content in the material, as evidenced by reduction in $\mathrm{Tg}$ and greater enthalpy of relaxation with increasing PAA concentration.
\end{abstract}

\section{Introduction}

Bloodmeal is a dried protein powder of red-brown colour produced by steam coagulating, decanting and rotary drying whole blood at $120{ }^{\circ} \mathrm{C}$. It contains about $95 \%$ protein, mainly haemoglobin and serum albumin and is an ideal feedstock for the production of bio-based plastics ${ }^{1}$. It can also be decoloured via chemical oxidation using commercial peracetic acid ${ }^{2}$. Peracetic acid is an equilibrium reaction product of hydrogen peroxide and ethanoic acid (acetic acid, AA) and sulphuric acid as catalyst ${ }^{3}$. The equilibrium composition is typically 5-6 wt\% peracetic acid (PAA), 21-23 wt\% hydrogen peroxide (HP), 10$11 \mathrm{wt} \%$ ethanoic acid and 63-65 wt $\%$ water ${ }^{4}$.

Due to the exposure of haemoglobin to the high temperatures employed during bloodmeal production, haem becomes trapped within the protein aggregates. The haem moiety in haemoglobin responsible for the dark colour can usually be degraded with an oxidant such as hydrogen peroxide or sodium hypochlorite 5-11 provided haem is freely soluble, easily accessible or is in the form of oxyhaemoglobin 6-8, 12. However, during bloodmeal production, exposure of haemoglobin to the thermal conditions employed causes significant structural changes to the protein ${ }^{13}$, 14 including conversion of oxyhaemoglobin to methaemoglobin 15 and aggregation into antiparallel $\beta$-sheets $13,14,16-19$. Consequently, hydrogen peroxide and sodium hypochlorite are no longer effective as decolouring agents ${ }^{6}$, and they are either unable to access a portion of the haem, or upon access, are unable to degrade all of the haem species present in bloodmeal 20. Thus the action of the peracetic acid on haem, specifically on methaemoglobin, is critical for obtaining adequate decolouring ${ }^{20}$.
An earlier model for decolouring bloodmeal proposed peracetic acid solution diffusing into bloodmeal particles leading to haem degradation, where all of the components of PAA play some role in the decolouring process ${ }^{20}$. Water and acetic acid led to swelling of the protein chains making them more mobile, and are thought to facilitate access of the oxidants to sites where they are able to react. In addition to haem degradation, peracetic acid and hydrogen peroxide are also likely to cause structural changes to the bloodmeal proteins during the diffusion process.

During decolouring, peracetic acid and hydrogen peroxide are undissociated due to the low $\mathrm{pH}$ of the system, and in their molecular form both act as electrophiles to attack sites high in electron density ${ }^{21}$. In this system, the amino acids most susceptible to oxidation are methionine and cysteine due to their high reactivity with electrophiles, and the aromatic amino acids tyrosine, tryptophan, phenylalanine and histidine due to their high electron density. These amino acids are commonly found in $\beta$-sheets and may be significantly changed upon decolouring with peracetic acid.

Oxidation is known to cause structural changes at a primary level including fragmentation, crosslinking and amino acid changes ${ }^{22}$. Upon oxidation with peracetic acid solutions, dairy proteins have been found to contain an increased number of carbonyl groups, reduced thiol (SH) groups, aggregated proteins and showed a reduced solubility at high PAA concentration ${ }^{23}$. Also, in contrast to bloodmeal, peracetic acid decoloured bloodmeal (DBM) has a glass transition temperature of around $50{ }^{\circ} \mathrm{C}$ and has a greater solubility in water and SDS solutions ${ }^{2}$. Solubility increased with the strength of PAA treatment, suggesting that hydrophobic 
interactions are reduced after PAA treatment. To successfully produce a bioplastic from DBM, only TEG and SDS were required, suggesting significant changes to protein structure, protein side groups, and/or protein-protein interactions have occurred during oxidation ${ }^{2}$.

The changes to primary structure caused by oxidation would also be expected to cause changes to the secondary structure of the protein due to the high dependence of secondary structure on the physicochemical and stereo-chemical properties of the amino acids of which they are comprised. Studies of the propensity of amino acids to form particular types of secondary structure indicate that most amino acids show preference for only one type of secondary structure ${ }^{24}$.

Typically, helical structures are comprised of amino acids containing short chain aliphatic side groups (Table 1). With the exception of alanine, all of these amino acids contain an aliphatic group on the $C \alpha$ carbon with no branching at the $C \beta$ position ${ }^{24-27}$. The amino acids with the highest propensity for $\beta$-sheets are most often non-polar or aromatic. Both threonine and cysteine are exceptions, threonine also tends to form bends and cysteine only has a slight propensity for sheets. The remaining amino acids are likely to form coils, bends or turns 24 .

Table 1. Propensity of amino acids for secondary structures types.

\begin{tabular}{ccc}
\hline Helical & Sheet & Other \\
\hline Alanine & Isoleucine & Glycine \\
Leucine & Phenylalanine & Proline \\
Methionine & Tryptophan & Asparagine \\
Glutamine & Valine & Aspartic acid \\
Glutamic acid & Tyrosine & Serine \\
Arginine & Threonine & Histidine \\
Lysine & Cysteine & \\
\hline
\end{tabular}

Prior to decolouring thermal drying of blood leads to the unravelling of $\alpha$-helices followed by the formation of intermolecular anti-parallel $\beta$-sheets responsible for aggregation 14. Understanding the secondary structural composition of bloodmeal and how it is altered by decolouring is important for the development of a useful material, as the physical properties of the polymer will determine how the protein should best be manipulated to obtain the desired material properties in the thermoplastic. Oxidation of native proteins with hydroxyl radicals has previously been found to cause change to the secondary and tertiary structures of bovine serum albumin (BSA), including denaturation and increased hydrophobicity, followed by the formation of intermolecular covalent bonding and increased proteolytic susceptibility ${ }^{28}$. The same study found that BSA oxidized by hydroxyl radicals in the presence of the superoxide anion and molecular oxygen caused denaturation followed by fragmentation at the $\alpha$-carbon and that the superoxide anion and molecular oxygen alone had no measurable change in secondary or tertiary structure. This suggests that the oxidant type and experimental conditions both determine the extent, if any, of secondary structural modification.
Synchrotron FTIR has recently been used to investigate secondary structure of agricultural proteins using the Amide I region 29,30 and of bloodmeal and its thermoplastic using the Amide III region ${ }^{31,32}$. It was found that although bloodmeal is comprised of denatured proteins, secondary structures such as $\beta$-sheets and $\alpha$-helices remain dispersed throughout random coil, amorphous regions ${ }^{32}$.

The aim of this work was to understand the effect of PAA treatment at various concentrations on protein secondary structure, chain mobility and changes in inter/intra molecular spacing of ordered regions. Secondary structural analysis was done by spatially resolving secondary structures using FTIR Synchrotron microscopy, wide angle X-ray scattering and differential scanning calorimetry.

\section{Experimental}

\section{Materials}

Agricultural grade bovine bloodmeal was obtained from Wallace Corporation Ltd, New Zealand and sieved to utilize particles under $710 \mu \mathrm{m}$. Peracetic acid (Proxitane Sanitizer 5\%) was purchased from Solvay Interox Pty Ltd Auckland, New Zealand and $30 \mathrm{wt} \%$ hydrogen peroxide from Sigma Aldrich, Castle Hill, NSW, Australia. Acetic and sulphuric acid and sodium hydroxide were purchased from Thermo Fisher Scientific, Auckland, New Zealand.

\section{Sample preparation}

Peracetic acid solution (300 g) was added to bloodmeal (100 g) and allowed to react with high speed mixing $(10 \mathrm{~min})$ to ensure homogenous decolouring before diluting with distilled water (300 g) a constant ratio of PAA solution to bloodmeal (3:1 w/w) was used. This mixture was immediately neutralized with sodium hydroxide and filtered. The decoloured bloodmeal was oven dried overnight $\left(75^{\circ} \mathrm{C}\right)$.

Decolouring BM with hydrogen peroxide (HP) solutions was carried out in the same ratio $(3: 1 \mathrm{w} / \mathrm{w}) \mathrm{HP}$ solution to BM, under the same reaction conditions. Upon completion the reaction was diluted with distilled water $(\sim 300 \mathrm{~g})$, filtered and oven dried overnight $\left(75^{\circ} \mathrm{C}\right)$.

To discern the effect of acetic acid on decolouring, bloodmeal (10 g) was decoloured using $26 \mathrm{wt} \%$ HP (30 g) containing $0.033 \mathrm{~mol}$ of acetic acid. After decolouring, the mixture was neutralized and oven dried overnight $\left(75^{\circ} \mathrm{C}\right)$. All samples were stored in air-tight polyethylene containers at ambient temperature for a minimum of one week prior to analysis.

\section{Instrumentation}

Spatially resolved FTIR experiments were undertaken on the infrared microspectroscopy beamline at the Australian Synchrotron, Victoria, Australia. Bloodmeal and decoloured bloodmeal particles were compressed in a diamond cell then transferred onto a barium fluoride slide. This was placed in a Linkam temperature controlled stage connected to a Bruker Hyperion 3000 with an MCT collector and XY stage. The 
stage was set to $24{ }^{\circ} \mathrm{C}$ and purged with nitrogen gas. For each sample type, three separate particles were mapped using a $10 \mathrm{x}$ $10 \mu \mathrm{m}$ spot size chosen on video images. For each point, 32 spectra were collected in transmission mode with a resolution of $4 \mathrm{~cm}-1$ between 3900 and $700 \mathrm{~cm}-1$ and averaged using Opus 6.5 software (Bruker Optik GmbH 2009).

The spectra with corresponding xy coordinates for each point on a particle were combined to give an overall spatial distribution for each particle and sample type. Relative peak height in the amide III region of inverted second derivative spectra was determined according to Bier et al ${ }^{31,32}$, and was used to estimate how relative fractional composition of corresponding secondary structures varied spatially across the particles after different decolouring treatments. Data was filtered for a minimum area under the amide III region to exclude points mapped outside particles from the analysis.

The second derivative of the original spectra (with no baseline correction) was determined using the Savitzky-Golay algorithm in Opus 6.5 using nine point smoothing. The second derivative was inverted by dividing by -1 and peak heights above the zero line were compared. The ratios $A_{\alpha} / A_{1}^{\prime \prime}, A_{t} / A_{\beta}^{\prime \prime}$ and $A_{r}^{\prime \prime} A_{\beta}^{\prime \prime}$ were calculated using Opus 6.5, where $A_{x}^{\beta}$ is the maximum ${ }^{\beta}$ height of the inverted second derivative peak within the wavenumber range associated with $\alpha$-helices, $\beta$-sheets, turns and random coils indicated respectively by the subscripts $\alpha, \beta, \mathrm{t}$ and $\mathrm{r}$. The molar fraction for each secondary structure type was calculated using Equations 1-5 and spatial maps were drawn based on these compositions using Microsoft Excel. The composition approximates a mole fraction of peptide linkages in each structural conformation, each of which absorbs differently in the amide III region (Table 2).

$$
\begin{aligned}
& \alpha+\beta+t+r=1 \\
& \beta=\frac{1}{A_{\alpha}^{\prime \prime} / A_{\beta}^{\prime \prime}+A_{t}^{\prime \prime} / A_{\beta}^{\prime \prime}+A_{r}^{\prime \prime} / A_{\beta}^{\prime \prime}+1} \\
& \alpha=\beta \frac{A_{\alpha}^{\prime \prime}}{A_{\beta}^{\prime \prime}} \\
& t=\beta \frac{A_{t}^{\prime \prime}}{A_{\beta}^{\prime \prime}} \\
& r=\beta \frac{A_{r}^{\prime \prime}}{A_{\beta}^{\prime \prime}}
\end{aligned}
$$

\begin{tabular}{ccc}
\hline Table 2: Peak assignment in the amide III region ${ }^{33}$. \\
\hline \multirow{2}{*}{ Region } & Secondary & Wavenumber/ cm- \\
& structure & 1 \\
\hline Amide III & $\alpha$-helix & $1330-1295$ \\
& $\beta$-turns & $1295-1270$ \\
& random coils & $1270-1250$ \\
& $\beta$-sheets & $1250-1220$ \\
\hline
\end{tabular}

Differential Scanning Calorimetry was conducted in a Perkin Elmer DSC 8500 hyper DSC fitted with an autosampler accessory and cooled with liquid nitrogen. Approximately 5-10 $\mathrm{mg}$ of sample was weighed into autosampler pans (Perkin Elmer) which were then crimped to provide a seal and placed into the auto sampler. All samples were scanned between 0 and $120{ }^{\circ} \mathrm{C}$ at $10^{\circ} \mathrm{C} / \mathrm{min}$ and cooled to $0{ }^{\circ} \mathrm{C}$ at the same rate prior to performing a second scan. Glass transition temperature (half $\mathrm{Cp}$ extrapolated) was determined using Pyris 7 software (Perkin Elmer). Excess enthalpy $(\Delta \mathrm{H})$ of relaxation was calculated by integrating the area under the endothermic peak, which occurred at the glass transition region, the reported values are means of two replicates

Wide angle X-ray scattering of decoloured samples was carried out at ambient temperature using a PANalytical Empyrean Xray diffractometer with a generator voltage of $45 \mathrm{kV}$ and a current of $40 \mathrm{~mA}$ using $\mathrm{CuK}^{\mathrm{a}}{ }^{1}$ radiation. The diffraction data was collected in the $2 \theta$ range from $4^{\circ}$ to $60^{\circ}$ with a step size of $0.105^{\circ}$. A soller slit of $0.04 \mathrm{rad}$ was used, with a fixed incident beam mask $15 \mathrm{~mm}$, a fixed $1^{\circ}$ anti-scatter silt and fixed $0.5^{\circ}$ divergence slit. For the diffracted beam path a fixed $7.5 \mathrm{~mm}$ anti-scatter slit was used and detected using a PIXcel3D area detector. Data was smoothed using a cubic function with 7point convolution in the X'Pert HighScorePlus software. A linear baseline was fitted to the minima between 4 and $60^{\circ}$ and subtracted and normalized to the peak occurring at $\sim 19^{\circ}$ in Excel. Peak position and d-spacing determined according to Bragg's equation were carried out in X'Pert HighScorePlus software.

\section{Statistical analysis}

For FTIR analysis, the average composition of each treatment type was calculated by averaging the secondary structure composition observed for each replicate and an analysis of variance with a two-tail Student's T-test was performed to identify significant changes ( $95 \%$ confidence) between the mean composition in BM and DBM samples. A summary of basic statistics for the distribution of secondary structures determined by FTIR microspectroscopy is available as supporting information.

\section{Results and Discussion}

Bloodmeal particles were found to be mostly heterogeneous, with $\beta$-sheets and helices randomly dispersed throughout (Table 3). Decolouring bloodmeal with HP or PAA led to changes to the average secondary structure composition of bloodmeal. The effect of PAA concentration on average composition is statistically significant, but relative to inherent structural variation it is only a small effect (Partial $\eta 2<0.1$ ), however the spatial distribution of secondary structures throughout the particle is dramatically changed upon PAA treatment.

Decolouring is a two phase reaction involving the diffusion of the oxidizing solution into the solid bloodmeal particle. The ease of diffusion is determined by concentration and also by the relative size and polarity of the diffusing species. PAA concentration was found to have a direct impact on the distribution of secondary structures due to the increasing content of the acetic acid and oxidants (Figure 1). The diffusion front is clearly observed as a change in secondary structure from the perimeter to the core of the particles, and is 


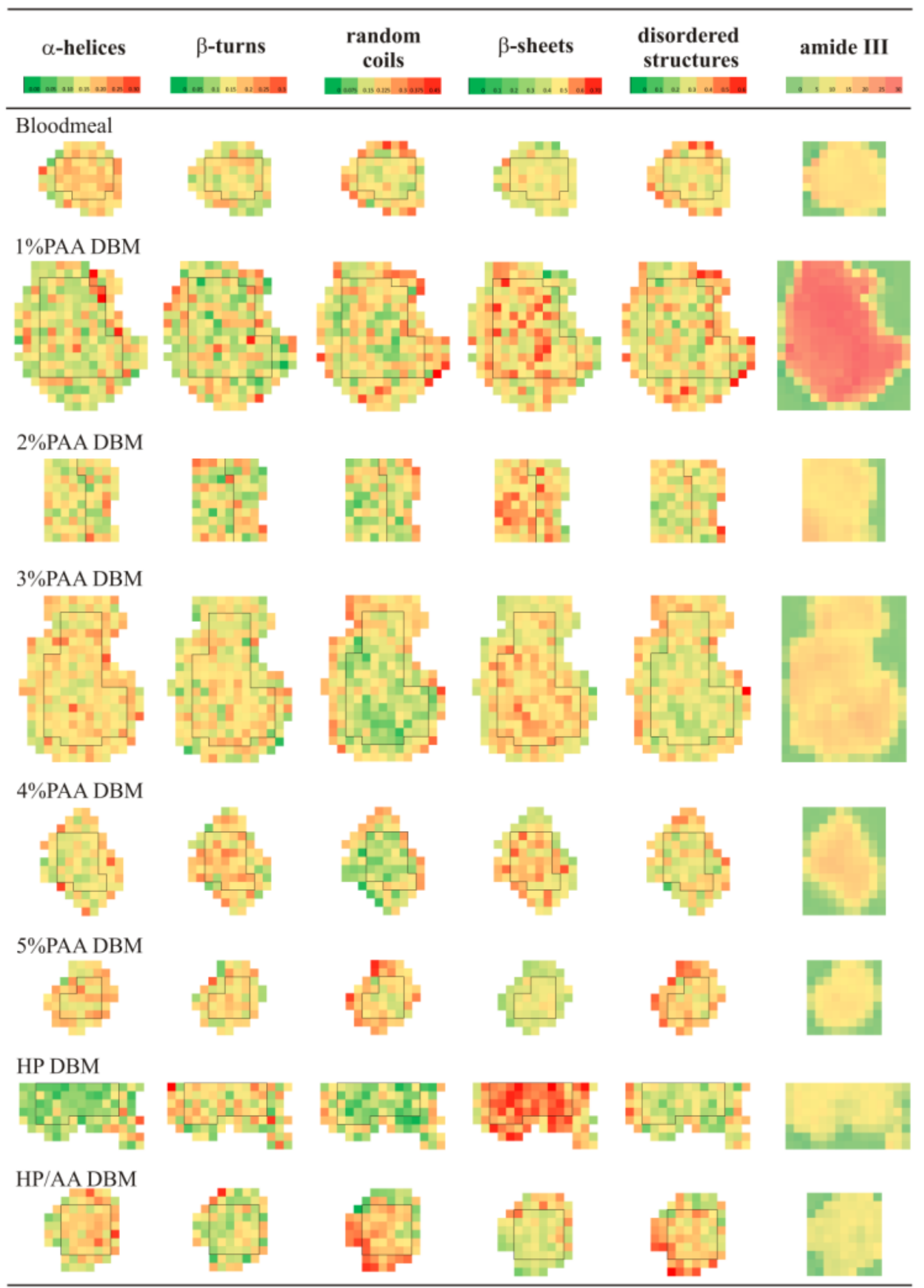

Figure 1. Secondary structure distribution and total amide III region of bloodmeal and decoloured bloodmeal (DBM) after oxidative decol ouring using peracetic acid (PAA), hydrogen peroxide (HP) or HP with acetic acid (HP/AA). Random coils and $\beta$-turns have been combined to give proportion of disordered structures. The shape outlined in black within the particle maps represents the boundary between the perimeter and the core of the particle. 
Table 3. Average fractional composition of secondary structures and one-way analysis of variance for decoloured bloodmeal, with PAA concentration as the independent variable. Partial $\mathrm{n}^{2}$ gives the effect size relative to inherent structural variation.

\begin{tabular}{lcccccc}
\hline Sample & Valid N & $\alpha$-helices & $\beta$-turns & random coils & $\beta$-sheets & disordered structures \\
\hline BM & 326 & $0.19 \pm 0.04$ & $0.15 \pm 0.04$ & $0.20 \pm 0.07$ & $0.46 \pm 0.06$ & $0.34 \pm 0.07$ \\
$1 \%$ PAA & 595 & $0.16 \pm 0.05$ & $0.15 \pm 0.06$ & $0.20 \pm 0.07$ & $0.49 \pm 0.09$ & $0.35 \pm 0.08$ \\
$2 \%$ PAA & 263 & $0.15 \pm 0.04$ & $0.15 \pm 0.05$ & $0.21 \pm 0.07$ & $0.50 \pm 0.08$ & $0.35 \pm 0.08$ \\
$3 \%$ PAA & 734 & $0.17 \pm 0.03$ & $0.15 \pm 0.04$ & $0.19 \pm 0.07$ & $0.49 \pm 0.06$ & $0.34 \pm 0.06$ \\
$4 \%$ PAA & 306 & $0.16 \pm 0.04$ & $0.18 \pm 0.05$ & $0.20 \pm 0.07$ & $0.46 \pm 0.08$ & $0.38 \pm 0.07$ \\
$5 \%$ PAA & 596 & $0.17 \pm 0.04$ & $0.18 \pm 0.05$ & $0.22 \pm 0.07$ & $0.43 \pm 0.06$ & $0.39 \pm 0.07$ \\
HP & 669 & $0.14 \pm 0.06$ & $0.17 \pm 0.06$ & $0.17 \pm 0.09$ & $0.52 \pm 0.11$ & $0.34 \pm 0.10$ \\
HP/AA & 591 & $0.17 \pm 0.04$ & $0.16 \pm 0.05$ & $0.23 \pm 0.07$ & $0.43 \pm 0.06$ & $0.40 \pm 0.07$ \\
Key results of one & way ANOVA versus PAA treatment concentration for each structure & & $<0.001$ \\
$p$ & $<0.001$ & $<0.001$ & $<0.001$ & $<0.001$ \\
Partial $\eta^{2}$ & 0.06 & 0.07 & 0.02 & 0.10 \\
\hline
\end{tabular}

$*$ Disordered structures $=\beta$-turns + random coils

particularly easy to discern in bloodmeal samples treated with solutions of 3-5 wt \% PAA.

Upon oxidation, some helical content is converted to other structures. Evaluating each treatment's secondary structure relative to bloodmeal indicated that hydrogen peroxide caused an overall reduction in helices and coils with a subsequent increase in sheets and turns (Figure 2). A slightly higher quantity of helices and turns were observed at the perimeter of the particle $(p<0.01)$. $\beta$-turns were probably formed in the highest quantity here because the surface of the particle is exposed to the highest concentration of HP; however it is unknown why helical structures remain in a higher concentration at the perimeter. The overall helical content was significantly reduced upon HP decolouring (Figure 2) and is most likely due to aggregation, leading to the observed increase in $\beta$-sheets, a phenomenon commonly observed in proteins exposed to high temperatures. The fact that HP is able to cause aggregation of helices and coils at the core of the bloodmeal particle indicates that HP readily diffuses throughout the particle. HP would presumably carry out the same function in peracetic acid solutions. In the presence of acetic acid, aggregation of helical structures occurred to a lesser extent, suggesting acetic acid may have a protective effect.

Additionally, the formation of $\beta$-sheets was suppressed compared with HP alone (Figure 2). Due to the suppression of helical aggregation and solvation of sheet structures with acetic acid, bloodmeal decoloured using HP and acetic acid mixtures resulted in increased structural disorder. The resulting particles showed regions of homogeneity (random coils and sheets) around the perimeter (Figure 1). However, as these regions corresponded to both the highest and lowest concentrations observed throughout the sample, they average out and the only conclusion which can be drawn in the case of HP containing acetic acid is that the particles were either agglomerated during decolouring and broke apart, or were affected by drying conditions leading to the observed contrast at the perimeter, compared with the core. Acetic acid therefore influences the resulting secondary structure, and also inhibits hydrogen peroxide consumption ${ }^{20}$.
However, it is uncertain whether the observed secondary structure is a result of the inhibition of oxidation, or the inhibition of oxidation is a result of the modification of the protein secondary structure in such a way that it sterically hinders access of HP to reactive sites. The role of acetic acid in PAA is probably similar, but due to its size may have greater difficulty diffusing through the protein compared with HP.

Decolouring with 1-3 wt\% PAA was found to cause aggregation of helices and random coils into sheet structures, with no change to $\beta$-turn content $(p>0.05)$. This change in composition was most strongly observed at the core of the particle, which is accessible to hydrogen peroxide (but not acetic acid due to its low concentration and larger size), whereas at the surface where acetic acid concentration would be highest, there was significantly less sheet formation (Figure 2). This effect became more pronounced at higher concentrations of PAA, until at $5 \mathrm{wt} \%$ PAA destruction of sheets at the core is also observed (Figure 1) with the formation of random coils, which could also be generated by protein chain scission ${ }^{2}$.

The highly reactive nature of both of the oxidants, suggest that it is unlikely the reaction occurs in a selective or systematic way, whereby helices and coils first become aggregated into sheets; that are subsequently degraded into turns and finally back into coils.

The behaviour of the decoloured bloodmeal material as a whole will be defined by the chemical properties of the proteins located throughout the particles. A definite difference in secondary structure composition exists between the perimeter and the core of the particle, caused by concentration gradient limited diffusion of acetic acid (Figure 3). The difference in the fractional composition of disordered structures ( $\beta$-turns and coils) between the perimeter and the core is greatest for 1-3 wt $\%$ PAA decoloured bloodmeal, and becomes reduced at 4-5 wt $\%$ PAA. 


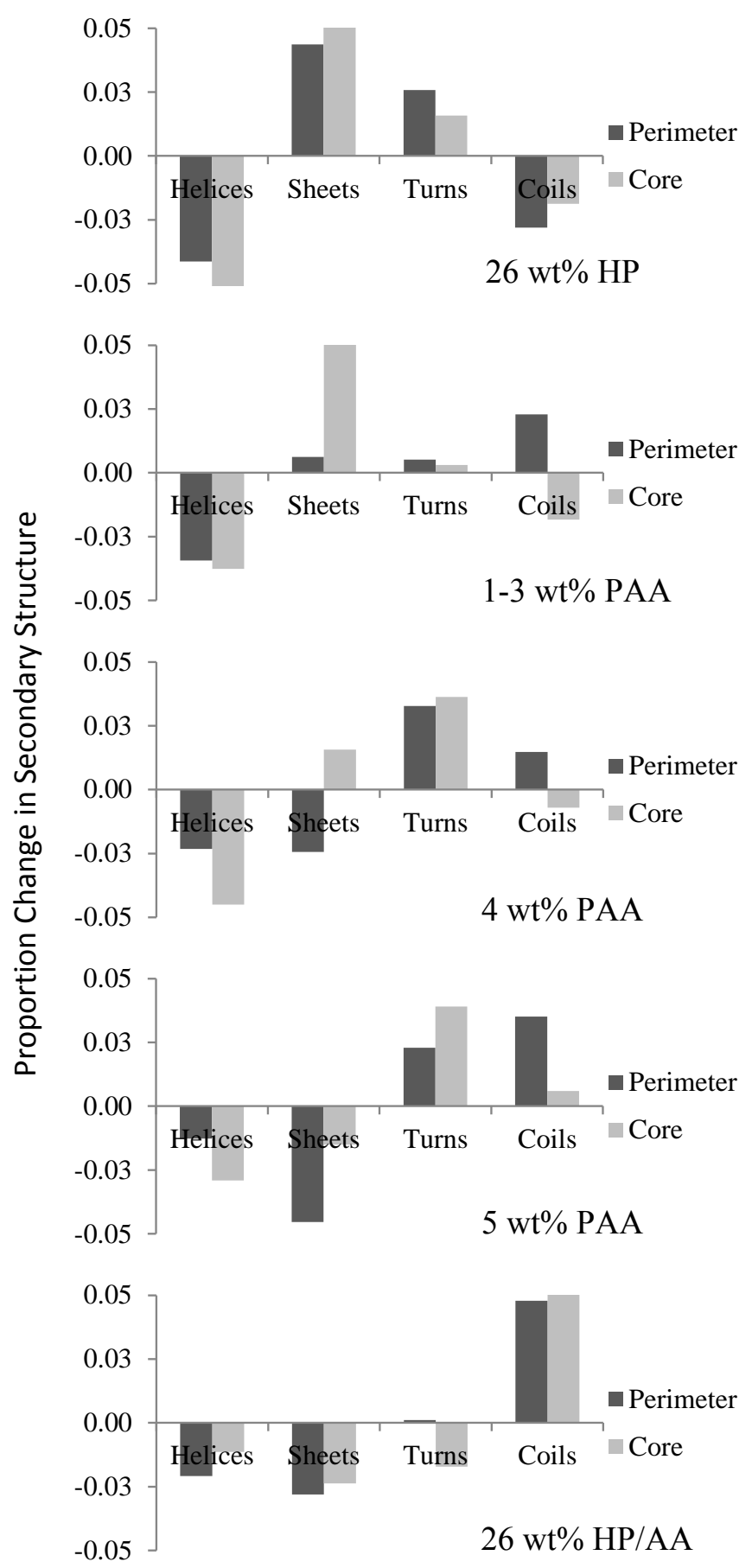

Figure 2. Change in secondary structure composition at both the perimeter and the core relative to bloodmeal. Top: $26 \mathrm{wt} \%$ hydrogen peroxide, 1-3 wt\%, 4 wt $\%$ and 5 wt $\%$ peracetic acid and bottom 26 wt $\%$ hydrogen peroxide with 6.6 $w t \%$ acetic acid.

At low PAA concentration, with limited diffusion, the properties of DBM are more likely to be representative of the structures found at the core of the particle and at higher PAA concentration the material is more likely to resemble the characteristics of the proteins located at the perimeter, as the perimeter would represent the greater mass.

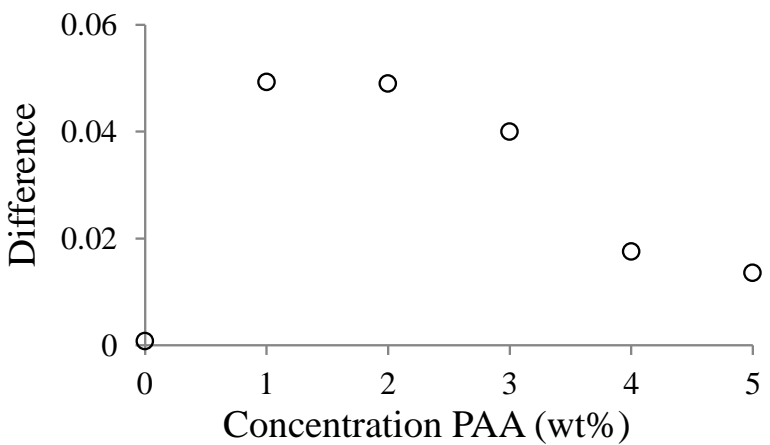

Figure 3. Change in fractional composition of disordered structures (turns and coils) between the perimeter and the core of the particles after decolouring with PAA.

Decolouring of bloodmeal with PAA was found to reduce the glass transition temperature from $\sim 225{ }^{\circ} \mathrm{C}$ to $\sim 35-45{ }^{\circ} \mathrm{C}$ using 3-5 wt $\% \mathrm{PAA}^{2}$. The glass transition temperature occurs when there is sufficient energy provided to obtain large scale cooperative motion within the amorphous regions of a polymer. In the case of a semi-crystalline material, both a glass transition temperature $(T g)$, at which the amorphous regions become mobile, and a higher melting temperature, where crystalline regions melt are observed ${ }^{34}$.

In bloodmeal, the $T g$ occurs above its degradation temperature $\left(\sim 200{ }^{\circ} \mathrm{C}\right)$ and decolouring with increasing concentrations of PAA resulted in a glass transition that is observed at progressively lower temperatures using DSC (Figure 4).

The rapid drop in $\mathrm{Tg}$ from $225^{\circ} \mathrm{C}$ to $80{ }^{\circ} \mathrm{C}$ after treatment with $1 \mathrm{wt} \%$ PAA indicates that oxidation has the strongest effect on $\mathrm{Tg}$, and its reduction is caused by the disruption of stabilizing interactions, specifically the destruction of crosslinks within the aggregated proteins leading to much higher chain mobility. The destruction of cysteine crosslinks during PAA decolouring allows DBM to be processed into a thermoplastic without the addition of sodium sulphite ${ }^{2}$. Acetic acid also improves chain mobility and can be observed as a continual, but lesser reduction in $T g$ with treatment of up to $5 \mathrm{wt} \%$ PAA. One could therefore conclude that the change in $T g$ is caused by a change in primary structure ie. chain mobility rather than the observed changes in secondary structure.

Bloodmeal and DBM showed an endothermic peak occurring below the glass transition temperature in the first DSC scans. In proteins, the helical and sheet structures undergo melting at temperatures exceeding $\sim 175^{\circ} \mathrm{C}$ at low moisture content ${ }^{35,} 36$, indicating the endothermic event observed is not associated with the melting of these crystalline structures. This endothermic peak is reported for numerous proteins and is thought to be caused by physical aging ${ }^{37-39}$, a phenomenon which occurs when an amorphous polymer is maintained below its glass transition temperature ${ }^{39}$. It is able to undergo aging because when it is rapidly cooled to a glassy state from above its $T g$, it is unable to physically reorient to an equilibrium conformation, causing the polymer to have a larger free volume, enthalpy and entropy than if it were in an equilibrium 
conformation ${ }^{40}$. As a result, the non-equilibrium material will undergo slow chain relaxation (through short-range, rotational reorientations) toward an equilibrium conformation ${ }^{41}$. The phenomena is dependent on time and the environment in which the polymer is maintained, where the extent of molecular relaxation is influenced largely by chain mobility and consequently increased temperature and plasticization (moisture content) during storage will cause a higher rate and extent of relaxation ${ }^{42}$.

When an aged polymer is heated above its $T g$, it absorbs the heat lost during aging and recovers its lost free volume and is accompanied by the development of an endothermic peak at its glass transition, observed during DSC ${ }^{39}$. In decoloured bloodmeal, the temperature at which this endothermic peak occurred was found to decrease with increasing PAA concentration used (Figure 5), indicating that less energy is required to mobilize the protein chains and is possibly a result of decreasing intermolecular interactions via oxidation and increasing acetic acid content providing plasticization.

The specific enthalpy of relaxation $(\Delta \mathrm{H})$ was also found to increase in size from 1-5 wt $\%$ PAA. Initially the specific enthalpy for bloodmeal was $0.69 \mathrm{~J} / \mathrm{g}$, and upon treatment with 1 wt $\%$ PAA increased to $1.4 \mathrm{~J} / \mathrm{g}$, up to $2.8 \mathrm{~J} / \mathrm{g}$ at 4 and $5 \mathrm{wt} \%$ PAA, all significantly lower than the heat of fusion associated with melting of helical or sheet crystallites. The increase in peak area reflects a larger degree of "aging" per gram of material taking place during drying and storage.

Oxidation is the primary cause of the increase in $\Delta \mathrm{H}$ by degrading ordered structures and destroying sterically hindering groups (such as aromatic amino acids) thereby improving chain regularity and allowing the protein chains to pack closer together. Due to their susceptibility to oxidation, it is expected that sulphur and aromatic amino acids, responsible for crosslinking and hydrophobic interactions within a protein chain, would be degraded. Oxidation of casein and whey proteins has been shown to cause formation of carbonyl compounds along with the destruction of tryptophan, methionine, tyrosine, histidine and lysine, and a decrease in free amino groups ${ }^{23}$. Destruction of these amino acids is also likely to occur during bloodmeal decolouring, leading to increased solubility ${ }^{2}$ and higher chain mobility as evidenced by the reduction in $T g$.

To a smaller extent, increasing acetate content may cause aging to occur more rapidly due to greater chain mobility, also facilitating closer packing. The effect of acetate ions on aging may become important when considering the final material produced from DBM, as aging is generally accompanied by a loss in mechanical properties due to lower reduced molecular mobility ${ }^{39}$.

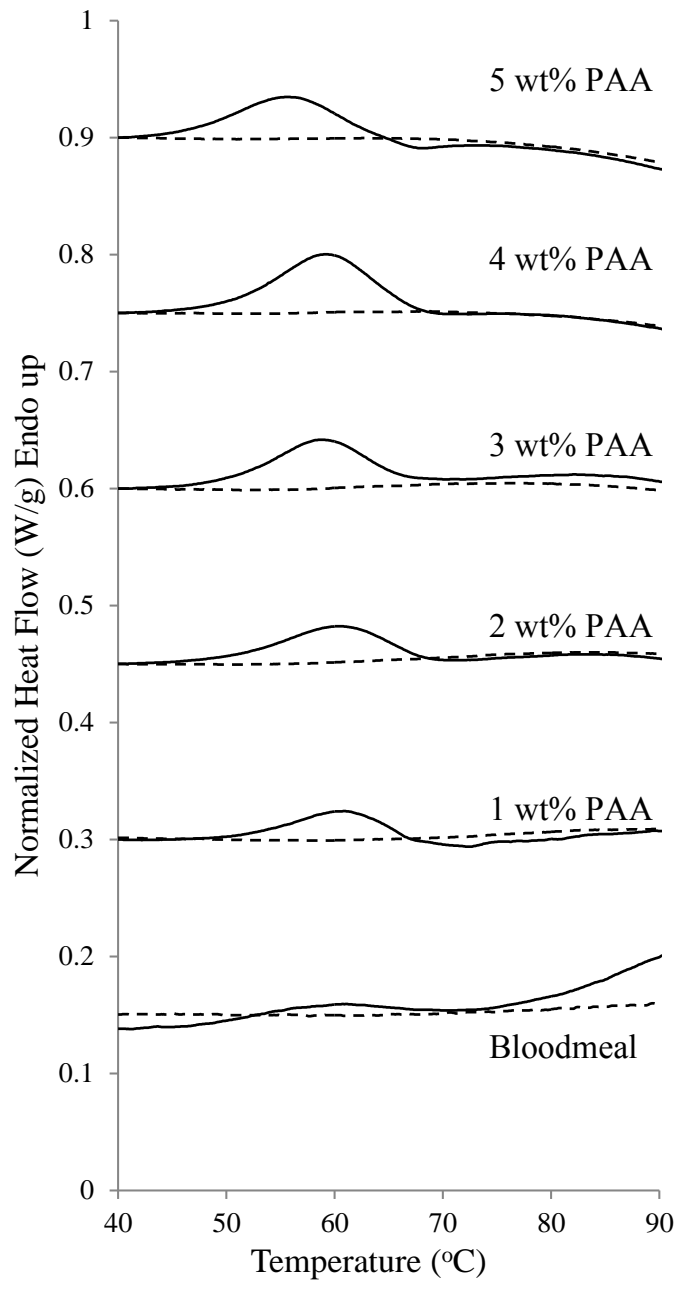

Figure 4. DSC thermograms of bloodmeal and its decoloured counterparts using a heating rate of $10^{\circ} \mathrm{Cmin}-1$. First scan solid line (-) and second scan dashed line (- -). Images have been stacked for clarity.

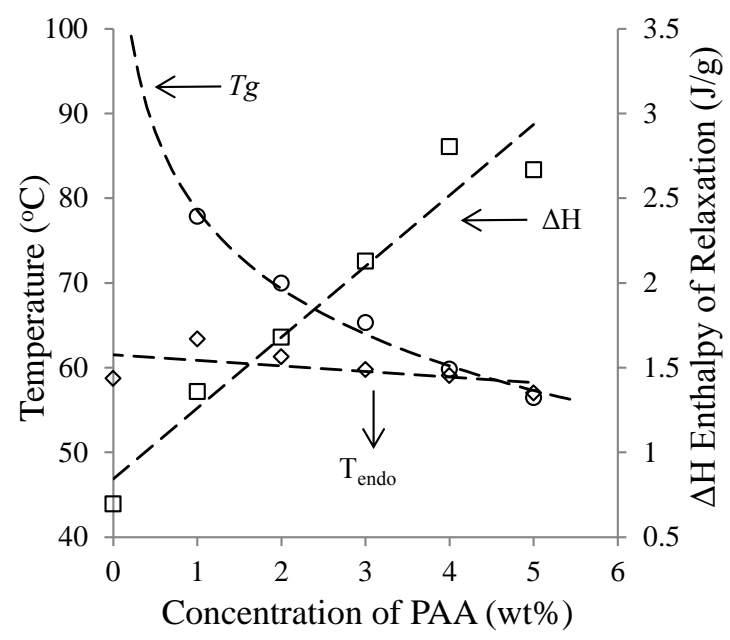

Figure 5. Thermal transitioning temperatures; glass transition, occurrence of the endothermic peak maximum and specific enthalpy of relaxation $(\Delta \mathrm{H})$ after bloodmeal decolouring with peracetic acid during DSC with a heating rate of 10 ${ }^{\circ} \mathrm{Cmin}-1$. Results are an average of duplicate samples. 
The shape of the X-ray diffraction curves for PAA treated bloodmeal are similar to that of untreated bloodmeal, with similar diffraction angles observed for each characteristic peak (Figure 6). For bloodmeal two main peaks were observed, one at $9^{\circ}$, corresponding to d-spacing of $9.9 \AA$, and an additional sharp peak at an angle of $19^{\circ}$ with a shoulder appearing at $24 \mathrm{o}$, corresponding to a d-spacing of $4.7 \AA$ and $3.8 \AA$ respectively. Peaks that correspond to these distances are often observed in proteins, and are thought to be attributable to $\alpha$-helix or $\beta$-sheet structures 43,44 . A d-spacing of 4-5 $\AA$ corresponds to interstrand hydrogen bonding in $\beta$-sheets or backbone hydrogen bonding in $\alpha$-helices, while a d-spacing of about $10.5 \AA$ corresponds to inter-structure packing either intersheet or interhelix spacing for proteins containing $\beta$-sheets or $\alpha$-helices 45 .

The peak observed at $9{ }^{\circ}$ was found at a slightly lower angle upon treating with 4-5 wt\% PAA. Such a shift could be indicative of larger spacing occurring between adjacent sheets or helices, however, it represents $\sim 0.5 \AA$ difference, and although changes in hydrogen bonding interactions between adjacent ordered structures could explain this shift, it may in fact be variation about a mean. The shoulder appearing at $\sim 24^{\circ}$ increased in breadth and height with 4-5 wt\% PAA concentration, also suggesting that destruction of ordered structures during oxidative decolouring leads to an increased amorphous content.

Equilibrium peracetic acid oxidants are responsible for breaking up intermolecular interactions between helical and random coil structures. Overcoming strong intermolecular interaction increases the mobility of these structures so that they are capable of rearranging into more highly aggregated sheet structures, most probably during drying $\left(\sim 75^{\circ} \mathrm{C}\right)$. Further to this, acetic acid is largely responsible for disrupting the process of aggregation; during decolouring or drying in the form of the acetate ion. Acetic acid and its conjugate base are both known to strongly hydrogen bond to amino acids found along the backbone of proteins, leading to a lower degree of secondary structural order, and results in a larger quantity of amorphous material being present along with increased chain mobility.

At high PAA concentration an additional peak develops at $\sim 7^{\circ}$, with a d-spacing of $12.6 \AA$. This peak is only observed in bloodmeal exposed to 4 or $5 \mathrm{wt} \%$ PAA, and is not present in samples which have been washed with water prior to drying and analysis. This peak may be an artefact of protein-acetate interactions but was not observed in bloodmeal mixed with sodium acetate salt or in bloodmeal pre-soaked in aqueous sodium acetate. However, the peak could originate as a result of separating adjacent sheets or helices apart by a further $\sim 2.7$ $\AA$ (compared with the main peak observed at $9.9 \AA$ ) through the incorporation of sodium or acetate ions between the structures of strongly oxidized structures. The only conclusion that can be drawn is that during decolouring with high concentration PAA, small water soluble compounds are generated, possibly due to salt-protein interaction or peptide chain scission and could influence the processing of DBM material into a thermoplastic in the future.
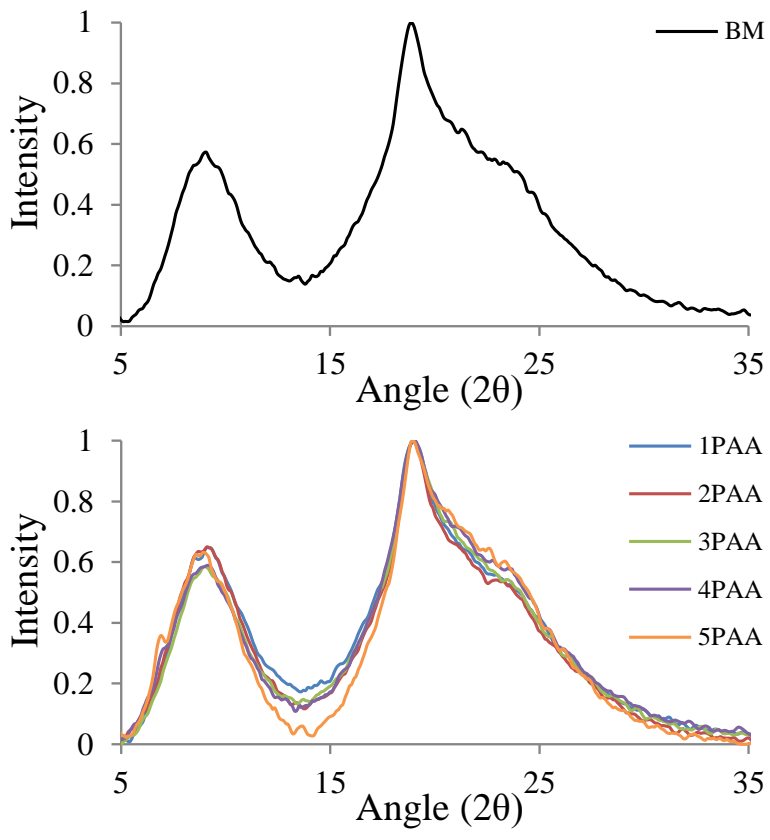

Figure 6. Normalized WAXS of bloodmeal (top) and 1-5 wt\% PAA treated bloodmeal (bottom).

\section{Conclusions}

Bloodmeal decolouring leads to two distinct regions of differing secondary structure composition resulting from diffusion of peracetic acid components into the particle. The innermost region of the particle is found to contain a high portion of $\beta$-sheets, owing to exposure to the oxidant species, most probably hydrogen peroxide, while the perimeter region contains much higher random coil content due to the degradation of ordered structures. The disordered structures are most concentrated at the perimeter due to the concentration of acetic acid and oxidant being highest at the surface of the particle.

Therefore PAA decolouring of bloodmeal can be classified into three distinct stages, where the final composition of the protein is dependent on the peracetic acid concentration employed. At low PAA concentration, $\alpha$-helices and random coils aggregate into $\beta$-sheet structures due to the presence of oxidants, at higher concentration the $\beta$-sheets begin to degrade into $\beta$-turns and at the highest concentration employed, degrade into coils due to increasing acetic acid and oxidant concentration.

Due to their susceptibility to oxidation, sulphur and aromatic amino acids responsible for crosslinking and hydrophobic interactions within a protein chain are degraded and lead to an increased solubility in water and SDS solutions, and higher chain mobility as evidenced by the reduction in $T g$ and improved mobility observed by the higher degree of aging with increasing PAA concentration. 


\section{Acknowledgements}

This research was undertaken on the infrared microspectroscopy beamline at the Australian Synchrotron, Victoria, Australia. Proposal number AS132/IRMFI/6636. The authors would especially like to acknowledge the technical assistance of Dr. Mark Tobin and Dr. Danielle Martin. Travel funding support was received from the New Zealand Synchrotron Group Ltd.

\section{Notes and references}

${ }^{a}$ School of Engineering, Faculty of Science and Engineering, University of Waikato, Private Bag 3105, Hamilton 3240, New Zealand

Electronic Supplementary Information (ESI) available: Basic statistics for distribution of secondary structures determined by FTIR microspectroscopy. See DOI: 10.1039/b000000x/

\section{References}

1. New Zealand Pat., 551531, 2010.

2. A. Low, C. J. R. Verbeek and M. C. Lay, Macromolecular Materials and Engineering, 2014, 299, 75-84.

3. B. Rangarajan, A. Havey, E. Grulke and P. Culnan, J. Am. Oil Chem. Soc., 1995, 72, 1161-1169.

4. FMC Corporation, Material safety data sheet: Peracetic acid 5\% 79-21-0-3, Philadelphia, 2006.

5. T. M. Florence, J. Inorg. Biochem., 1985, 23, 131-141

6. A. Low, Ph.D. Thesis, University of Waikato, 2012

$7 . \quad$ A. Low, J. Verbeek, M. Lay and J. Swan, Biochem. Biotechnol., 2012, 42, 29-43.

8. E. Nagababu and J. M. Rifkind, Biochem. Biophys. Res. Commun., 1998, 247, 592-596.

9. E. Nagababu and J. M. Rifkind, Biochem. Biophys. Res. Commun., 2000, 273, 839-845.

10. E. Nagababu and J. M. Rifkind, Antioxid. Redox Signaling, 2004, 6. $967-978$

$11 . \quad$ U.S.A. Pat., $4650589,1987$.

12. E. Nagababu and J. M. Rifkind, Biochemistry, 2000, 39, 12503 12511 .

13. Y. Seto, M. Kataoka and K. Tsuge, Forensic Sci. Int., 2001, 121, 144-150.

14. Y. Yan, Q. Wang, H. He and H. Zhou, Biophys. J., 2004, 86, 1682-1690.

15. P. Labrude, B. Chaillot and C. Vigneron, Cryobiology, 1984, 21, 33-38.

16. J. F. Black and J. K. Barton, Photochem. Photobiol., 2004, 80, 8997.

17. J. F. Black, N. Wade and J. K. Barton, Lasers Surg. Med., 2005 , 36, 155-165.

18. H. Jansson and J. Swenson, J. Chem. Phys., 2008, 128, 245104

19. C. Liu, A. Bo, G. Cheng, X. Lin and S. Dong, Biochim. Biophys. Acta, Protein Struct. Mol. Enzymol., 1998, 1385, 53-60.

$20 . \quad$ T. M. Hicks, C. J. R. Verbeek, M. C. Lay and M. Manley-Harris, $J$ Am Oil Chem Soc, 2013, 1-11.

21. M. Unis, Ph.D. Thesis, University of Northumbria, 2010.

22. W. Zhang, S. Xiao and D. U. Ahn, Crit. Rev. Food Sci. Nutr., 2013, 53, 1191-1201.

23. B. Kerkaert, F. Mestdagh, T. Cucu, P. R. Aedo, S. Y. Ling and B. De Meulenaer, J. Agric. Food Chem., 2011, 59, 907-914.

24. S. Malkov, M. Živković, M. Beljanski, M. Hall and S. Zarić, $J$. Mol. Model, 2008, 14, 769-775.

25. P. Y. Chou and G. D. Fasman, Biochemistry, 1974, 13, 211-222.
S. Costantini, G. Colonna and A. M. Facchiano, Biochem. Biophys. Res. Commun., 2006, 342, 441-451.

M. Levitt, Biochemistry, 1978, 17, 4277-4285.

K. J. Davies and M. E. Delsignore, Journal of Biological Chemistry, 1987, 262, 9908-9913.

P. Yu, Br. J. Nutr., 2004, 92, 869-885.

P. Yu, J. J. McKinnon, C. R. Christensen and D. A. Christensen, J. Agric. Food Chem., 2004, 52, 7353-7361.

J. Bier, C. Verbeek and M. Lay, J. Therm. Anal. Calorim., 2013, $1-9$.

J. M. Bier, C. J. R. Verbeek and M. C. Lay, J. Appl. Polym. Sci., 2013, 130, 359-369.

S. Cai and B. R. Singh, Biophys. Chem., 1999, 80, 7-20.

J. M. Bier, C. J. R. Verbeek and M. C. Lay, Macromol. Mater. Eng., 2013, n/a-n/a.

J. Cao, Thermochim. Acta, 1999, 335, 5-9.

X. Hu, Q. Lu, D. L. Kaplan and P. Cebe, Macromolecules, 2009, 42, 2079-2087.

C. Bengoechea, A. Arrachid, A. Guerrero, S. E. Hill and J. R. Mitchell, J. Cereal Sci., 2007, 45, 275-284.

A. Farahnaky, F. Badii, I. A. Farhat, J. R. Mitchell and S. E. Hill, Biopolymers, 2005, 78, 69-77.

X. Mo and X. Sun, J. Polym. Environ., 2003, 11, 15-22.

J. W. Lawton and Y. V. Wu, Cereal Chemistry, 1993, 70, 471475.

L. C. E. Struik, Physical aging in amorphous polymers and other materials, Elsevier, Amsterdam, 1978.

F. Badii, W. MacNaughtan and I. A. Farhat, Int. J. Biol. Macromol., 2005, 36, 263-269.

C. S. Thomas, M. J. Glassman and B. D. Olsen, ACS Nano, 2011, 5, 5697-5707.

Y. Wang, F. L. Filho, P. Geil and G. W. Padua, Macromol. Biosci., 2005, 5, 1200-1208.

W. Elshemey, A. Elfiky and W. Gawad, Protein J, 2010, 29, 545550 . 